\title{
Aplicación del Modelo Canvas para el mejoramiento administrativo de una comercializadora de lubricantes para automotores
}

\author{
Julio Cesar Montoya R. ${ }^{1}$ \\ Henry Hurtado Bolaños ${ }^{2}$ \\ Alicia Cristina Silva C. ${ }^{3}$
}

\section{RESUMEN}

El presente proyecto tuvo como intencionalidad práctica aplicar el Modelo Canvas para una Lubriteca palmirana, para este fin lo primero que se hizo, fue contextualizar el tema objeto de estudio y ajustar esta metodología a las características específicas de la empresa; en relación a sus ámbitos endógeno y exógeno de la comercializadora, con el objeto de determinar la realidad situacional de este negocio. En esta parte puntualmente se efectuó un proceso de análisis por medio de la herramienta analítica DOFA.

En segunda instancia, se procedió a aplicar cada uno de los bloques estructurales de este modelo, o sea resolver cada uno de los ítems que lo conforman, con base en la información específica de la Comercializadora.

La parte definitiva de esta investigación, correspondió a manera de colofón, a la socialización de los potenciales resultados de la aplicación de este modelo de negocio con la Gerencia de la Comercializadora y su talento humano, para definir los lineamientos administrativos a seguir.(actividades, tareas, procedimientos, roles, funciones, etc.).

\footnotetext{
${ }_{1}^{1}$ Administrador de Empresas, Especialista en Pedagogía para el Desarrollo del Aprendizaje Autónomo, Maestría en Administración de Empresas. Docente de la Universidad Nacional Abierta y a Distancia UNAD. julio.montoya@unad.edu.co

${ }^{2}$ Administrador de Empresas, Especialista en Administración de Salud, Magíster en Ciencias de la Organización, profesor Universidad Nacional Abierta y a Distancia - UNAD. henry.hurtado@unad.edu.co ${ }^{3}$ Administradora de empresas, especialista en Pedagogía para el Desarrollo del Aprendizaje Autónomo. Magíster en Sistemas de Calidad y Productividad.Estudiante de Doctorado de Administración de Negocios, mención Gerencia. Docente Universidad Nacional Abierta y Distancia - UNAD. alicia.silva@unad.edu.co.
} 
Palabras clave: Propuesta de valor, actividades clave, relaciones con el consumidor, mercado meta, recursos, socios, estructura de costos

\section{Introducción}

Las organizaciones de vanguardia propenden día a día por identificar elementos diferenciadores que les permitan posicionarme mejor con sus clientes, la idea es crear valor en sus procesos, en sus prácticas, en sus productos, en su forma de servir, en lo que se encuentre al alcance, con tal de conquistar el exigente interés de los consumidores.

Los modelos de negocio nacieron como consecuencia de esta necesidad, y los que se dedican a investigar las multiformes maneras de hacer negocios rentables en un contexto globalizado, son conscientes de que lo que realmente funciona es "agregar valor al cliente".

Una opción de modelo de negocio amigable es llamado "CANVAS"; que precisamente surgió ante la necesidad de guiar a los emprendedores hacia el éxito. Canvas es una herramienta que permite visualizar de manera global todos los aspectos que configuran la empresa y el modelo de negocio, y realizar modificaciones en cualquier momento a medida q se va avanzando en el análisis. (Osterwalder \& Pigneur, 2011).

La presente investigación académica, plantea la aplicación de este modelo para lograr mayor eficacia en los ingresos de la una comercializadora de lubricantes para automotores, asentada en el municipio de Palmira, Valle.

\section{Marco Teórico}

\section{Que es el modelo CANVAS?}

EI CANVAS es una herramienta para confeccionar modelos de negocio propuesta por Alexander Osterwalder donde describe de manera lógica la forma en que las organizaciones crean, entregan y capturan valor. (Osterwalder \& Pigneur, 2011). El proceso del diseño del modelo de negocio es parte de la estrategia de éste, por lo que es de vital importancia conocer en profundidad cómo opera la empresa y al mismo tiempo sus fortalezas y debilidades. El modelo CANVAS es una herramienta que nos debe permitir confeccionar nuestro propio modelo de negocio y ayudarnos a validar 
su viabilidad, no sólo económica, sino de planteamiento de todos los elementos en el entorno de la empresa o proyecto empresarial.

Ilustración 1. Cuatro preguntas básicas del modelo Canvas
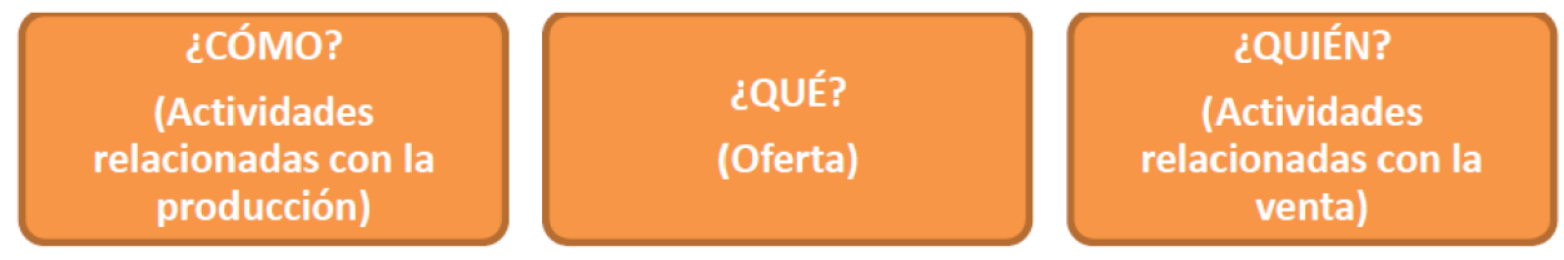

¿£?

(Finanzas)

¿£?

La ilustración anterior se basa en un diagrama denominado CANVAS, el cual parte de cuatro (4) preguntas básicas que reflejan 4 áreas principales que configuran la primera armadura del modelo de negocio del proyecto empresarial:

Ante todo, lo que se debe tener claro, para cualquier empresa que se apoye en el Canvas, es saber cuál es nuestra propuesta de valor (¿QUÉ?), A qué clientes la dirigimos (¿QUIÉN?) Y qué tenemos para hacerlo (¿COMO?). Finalmente, esbozaremos los recursos económicos que obtendremos los clientes y los gastos que nos supondrá elaborar la propuesta de valor.

\section{Los 9 módulos del Modelo Canvas}

El modelo CANVAS lo conforman nueve bloques que desarrollan las cuestiones planteadas inicialmente y que nos ayudará a validar la forma de hacer viable el proyecto empresarial (Osterwalder \& Pigneur, 2011)

Los bloques son:

1. Segmentos de clientes: los diferentes grupos de personas o entidades a las que dirigimos las propuestas de valor. Para quien creamos valor? Nos dirigimos a uno o a diferentes segmentos? (Mercado de masas, nicho de mercado, mercado segmentado ...)

2. Propuestas de valor: el conjunto de productos y servicios que crean valor para un segmento de mercado específico. El objetivo es solucionar los problemas de los clientes y satisfacer sus necesidades mediante propuestas de valor. ¿Qué problema ayudamos a solucionar? ¿Qué valor ofrecemos a nuestros clientes? Hay que plantearlo desde la perspectiva de "qué quiere comprar nuestro cliente" versus "qué vendemos". 3. Canales de comunicación, distribución y venta: la forma en que la empresa establece contacto con los diferentes clientes y cómo les proporciona la propuesta de valor. 
4. Relación con los clientes: los diferentes tipos de relaciones de la empresa con cada segmento de clientes, que establecen y mantienen de forma independiente para cada segmento. En función de cada cliente, adaptaremos el discurso.

5. Ingresos: se generan cuando los clientes adquieren las propuestas de valor que ofrece la empresa.

6. Recursos y capacidades clave: los activos necesarios para el modelo de negocio, incluidos las personas de la empresa y sus capacidades.

7. Actividades clave: las acciones necesarias que deben llevarse a cabo. Habrá que saber si contamos con las capacidades necesarias (y recursos clave) para llevar a cabo estas actividades.

8. Alianzas clave: las alianzas, los socios, incluso los proveedores que necesitamos para el éxito del modelo de negocio. Quizá algunas actividades se pueden externalizar y / o determinados recursos se pueden adquirir fuera de la empresa.

9. Estructura de Costos: toda la puesta en marcha de un negocio para poder elaborar y hacer llegar la propuesta de valor a los clientes tiene unos costes asociados. ¿Cuál es la estructura de costes?

Ilustración 2. Nuevo módulos de la herramienta CANVAS

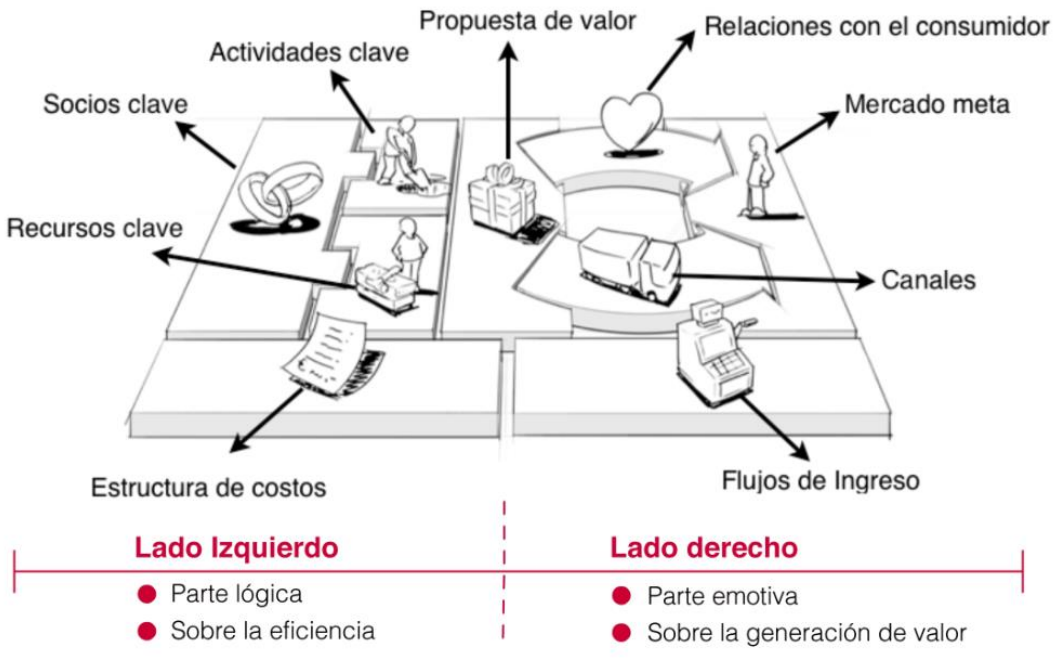

\section{Ventajas del Canvas}

- Permite ver el modelo de forma resumida en una sola página y nos ayuda a diseñar e innovar.

- Ayuda al pensamiento estratégico y a entender la situación real de la empresa ofreciendo una visión de conjunto con una visión a alto nivel del modelo de negocio.

- Permite experimentar en distintos escenarios, lo que facilita la toma de decisiones.

- Se integra muy bien con otras herramientas del nuevo management, como Customer Development, Lean Startup, Estrategia de Océanos azules, etc.

- Fomenta el trabajo colaborativo 


\section{Operacionalizacion del Modelo Canvas (Lubricantes S.A)}

Una vez contextualizado tanto la empresa en sus entornos exógeno y endógeno se procedió a operacionalizar el Modelo Canvas a la especificidad de la Pyme (Lubricantes Vega)

\section{Ilustración 3. Esquema estructural modelo Canvas}

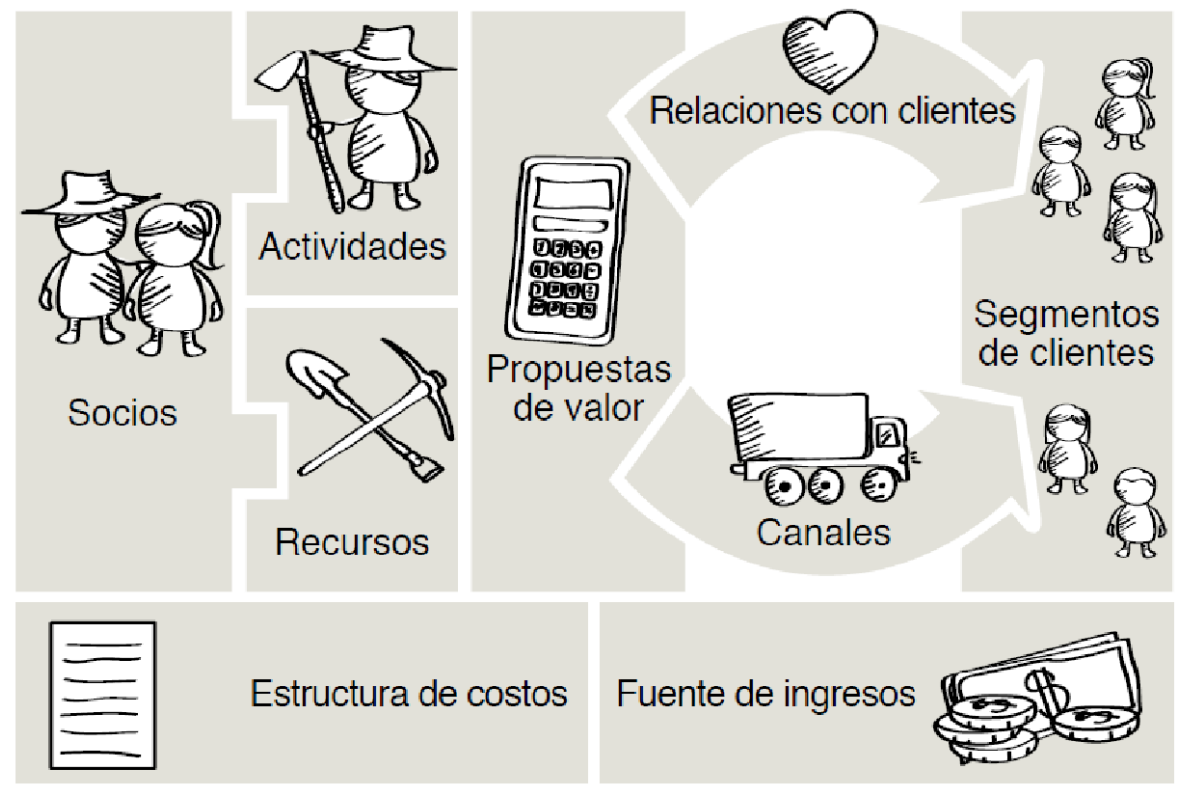

Fuente: Mycoop Colombia

El esquema anterior, resume los nueve (9) bloques que conforman el modelo Canvas, tiene como punto de partida la segmentación de clientes, y como punto final la fuente de ingresos.

\section{Bloque 1. Segmentación de Clientes}

En este primer bloque se definen uno o varios segmentos de mercado que la organización se va a dirigir, basado en fundamentos de conocimiento de los mismos (necesidades, comportamiento o atributos comunes) y del servicio o producto que está ofreciendo. Estos pueden ser pequeños o grandes segmentos. 
Ilustración 4. Segmentación de clientes

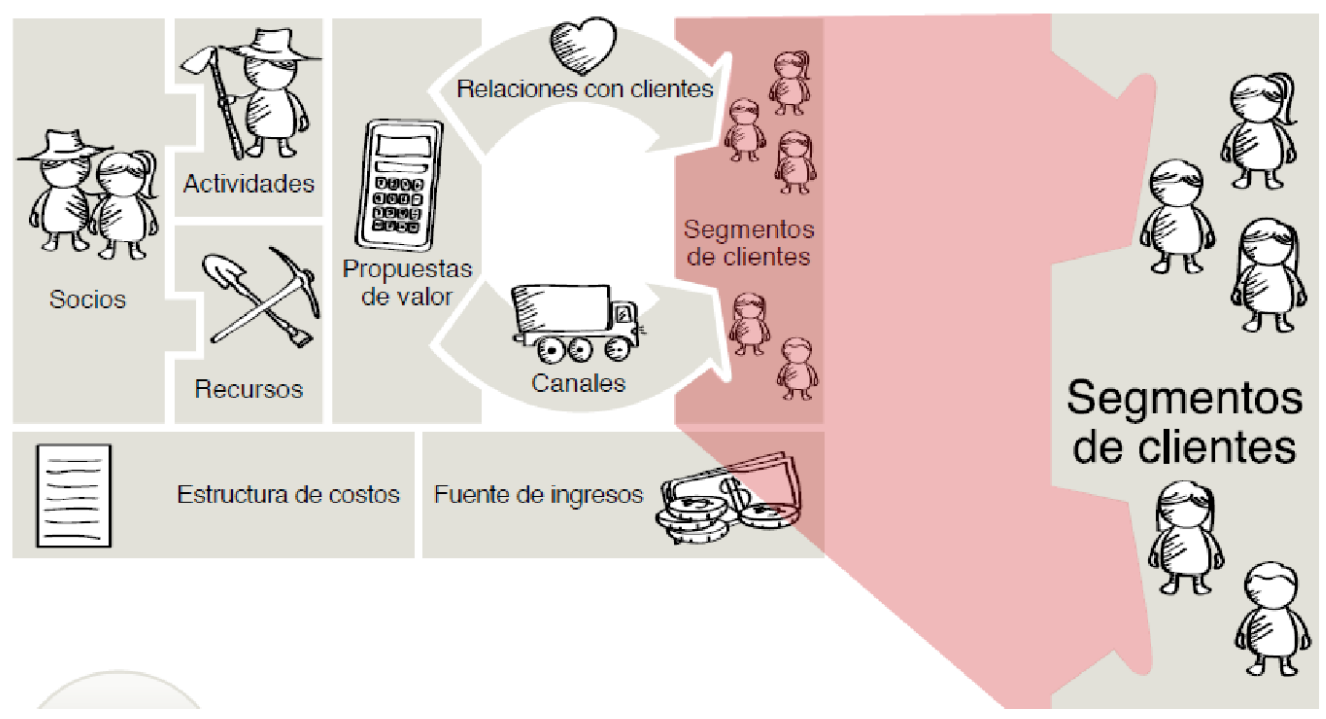

Fuente: Mycoop Colombia

\section{Preguntas relacionadas con sus respectivas respuestas}

\begin{tabular}{ll}
\hline Preguntas & $\begin{array}{l}\text { Respuestas para la Pyme (Lubricantes Vega } \\
\text { S.A.S }\end{array}$ \\
\hline Para quién estamos creando valor? & $\begin{array}{l}\text { La empresa crea valor para sus innumerables } \\
\text { clientes dueños de automotores (tanto de } \\
\text { vehículos como motocicletas) por medio de la } \\
\text { oferta de un gran surtido y un servicio al cliente } \\
\text { teniendo como elemento diferenciador el servicio } \\
\text { domiciliario de cambio de aceite }\end{array}$ \\
$\begin{array}{l}\text { Quiénes son nuestros clientes principales de Lubricantes Vega } \\
\text { importantes? }\end{array}$ & $\begin{array}{l}\text { S.A.S son los dueños de vehículos (carros) que } \\
\text { visitan la Lubriteca para procesos de lubricación y } \\
\text { cambios de aceite }\end{array}$ \\
\end{tabular}

\section{Segmento de clientes}


- Estrato socioeconómico: 2 -3-4-5

- Hombres y mujeres mayores de edad, propietarios de automotores (vehículos, camperos, camionetas, busetas pequeñas, motocicletas)

- Edades entre 18 y 55 años de edad

- Empleados, comerciantes, independientes, operarios, obreros, taxistas, motoristas automotores pequeños, que viven o tienen paso obligado por el sector Norte de la ciudad.

- Mercado de masas (son productos y servicios que sirven para todo público que posea vehículos automotores hasta de dos ejes.

\section{Bloque 2. Propuesta De Valor}

Partiendo de la base que el bloque de segmento de clientes es el centro de un modelo de negocios, puede decirse que la propuesta de valor es el corazón del mismo, dado que es un conjunto de productos y servicios que crean valor para un segmento de mercado especifico de acuerdo a las necesidades de dicho segmento. (Matiz, 2014)

\section{Ilustración 5. Propuesta de valor}

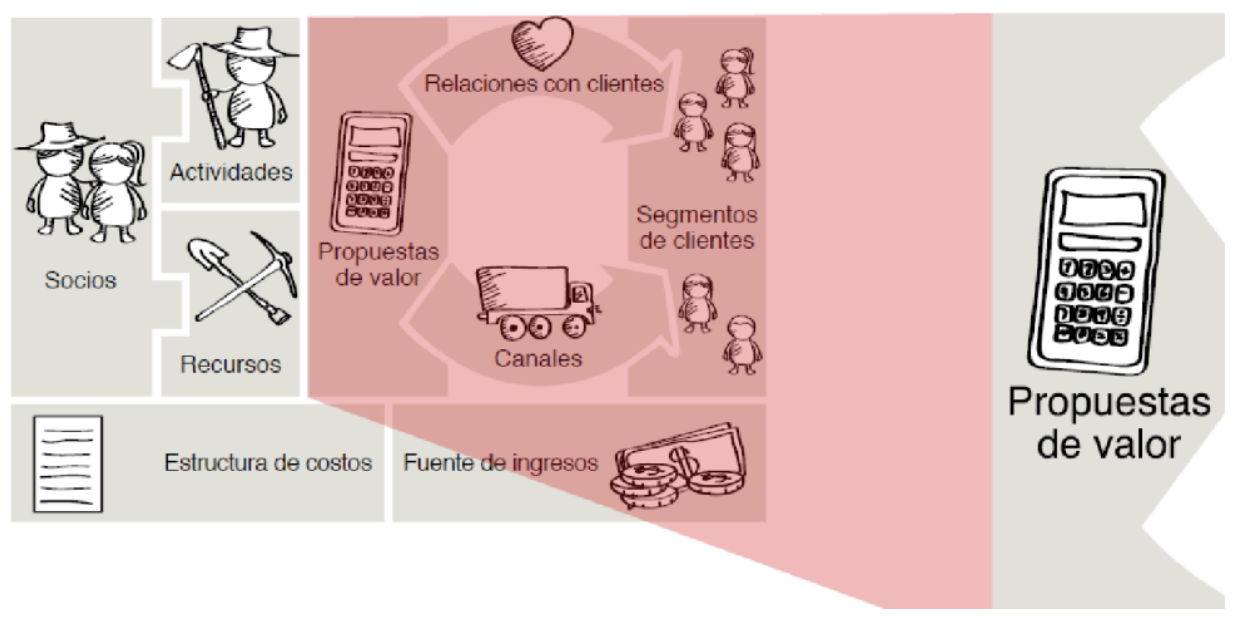

Fuente: Mycoop Colombia

\section{Preguntas relacionadas con sus respectivas respuestas}

\begin{tabular}{ll}
\hline Preguntas & $\begin{array}{l}\text { Respuestas para la Pyme (Lubricantes Vega } \\
\text { S.A.S }\end{array}$ \\
\hline Qué ofrezco y por qué me prefieren? & $\begin{array}{l}\text { Lubricantes Vega S.A.S ofrece productos de } \\
\text { marca, y un servicio diferenciado de lubricación y } \\
\text { cambio de aceite; los clientes prefieren esta }\end{array}$ \\
lubriteca, porque la atención es óptima y \\
oportuna.
\end{tabular}


Cuál de los problemas de nuestro cliente estamos solucionando?

Qué necesidad estamos satisfaciendo?
La empresa en este momento se encuentra optimizando la gestión del servicio domiciliario para mitigar las quejas asociadas con la prestación de este servicio.

La necesidad de los clientes de tener un automotor en condiciones óptimas de lubricación.

\section{Propuesta de valor para Lubricantes Vega : "BUENA SALUD PARA TU AUTOMOTOR"}

\section{Bloque 3. Canales}

Continuando con el análisis de los bloques de la herramienta propuesta por Osterwalder, entramos en el bloque de canales. Los canales son la forma en que se entregan los beneficios por medio de distribución, comunicación, ventas y canales estratégicos eficientes que logren su cometido; la manera en que mi empresa se comunica con los clientes para llevar mi propuesta de valor a los segmentos de clientes que he definido. (Osterwalder \& Pigneur, 2011)

Ilustración 6. Canales

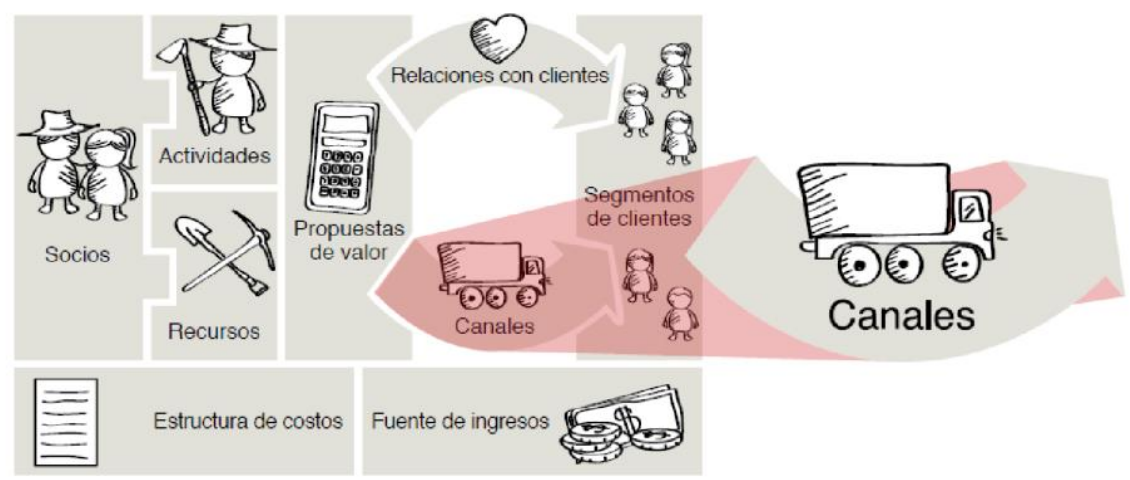

Fuente: Mycoop Colombia

\section{Preguntas relacionadas con sus respectivas respuestas}

\begin{tabular}{ll}
\hline Preguntas & Respuestas para la Pyme (Lubricantes Vega S.A.S \\
\hline ¿Qué canales prefieren nuestros segmentos & Los clientes de Lubricantes Vega S.A.S prefieren el canal
\end{tabular}


de mercado?

¿Cómo establecemos actualmente el contacto con los asociados y clientes?

¿Cuáles tienen mejores resultados?

¿Cuáles son los más rentables?

¿Cómo nos integramos o adecuamos a las actividades de los clientes? indirecto-tienda, donde ellos pueden visitar nuestro local $y$ adquirir los lubricantes e insumos asociados

Por la naturaleza comercial de esta Pyme, los clientes suelen visitar el negocio para recibir el servicio de lubricación, o en su defecto arriman al local a adquirir insumos, aparte de este contacto directo, se establece comunicación con ellos mediante una base de datos de clientes y se les informa vía e-mail, sobre nuevos productos y promociones

La interacción directa con ellos

Los canales más rentables o más eficaces son los directos

Los procesos de integración son informales

Ilustración 7. Tipos de canales

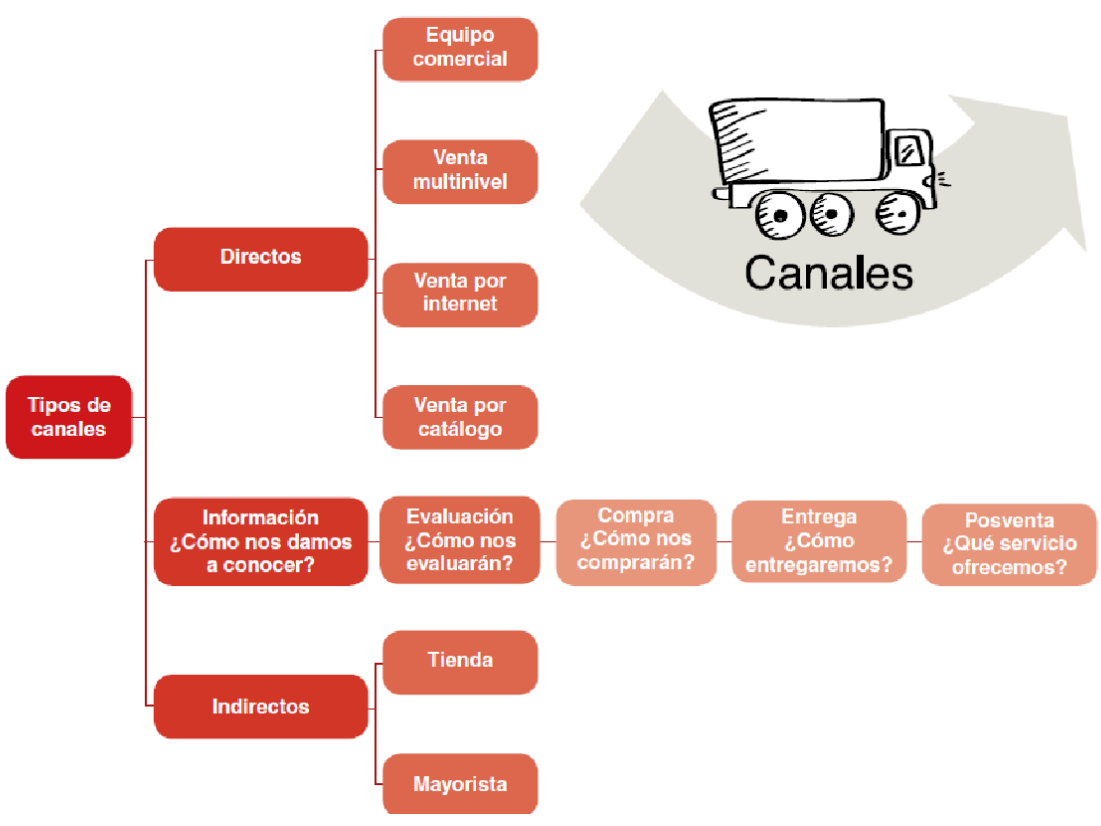

Fuente: Mycoop Colombia

\section{Tipos de canales utilizados por Lubricantes Vega}

- Directos: Contacto por internet (vía e-mail)

- Indirectos: Tienda (local)

\section{Bloque 4. Relaciones Con Los Clientes}


Para finalizar los bloques del segmento "para quién?" de la llustración 5, o sea, los quetienen que ver con los clientes, tenemos el bloque de la "relación con ellos".Es necesario definir qué tipo de relación desea tener con cada segmento de clientes que puede ser de captación, fidelización o estimulación de las ventas.

Ilustración 8. Relaciones con los clientes

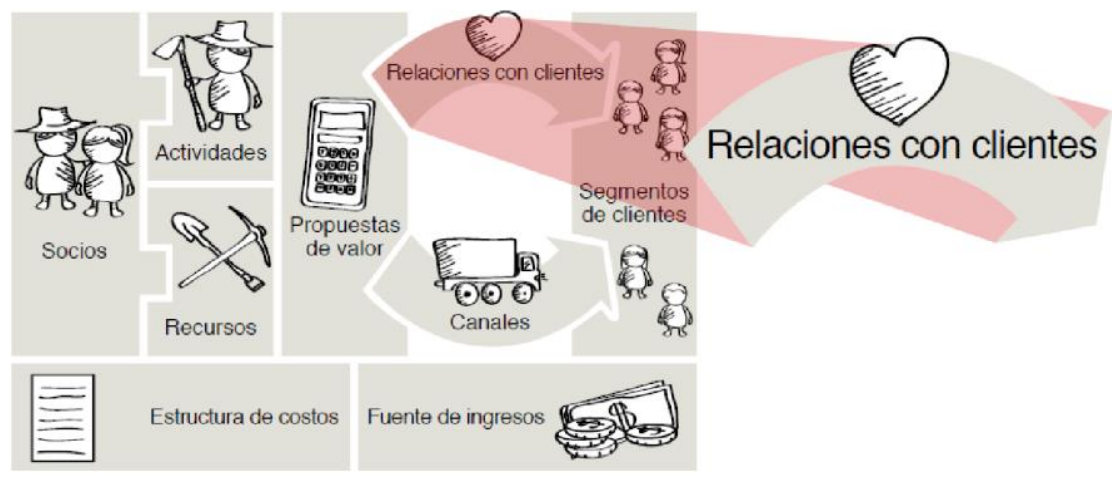

\section{Preguntas relacionadas con sus respectivas respuestas}

\begin{tabular}{ll}
\hline Preguntas & $\begin{array}{l}\text { Respuestas para la Pyme (Lubricantes Vega } \\
\text { S.A.S }\end{array}$ \\
\hline $\begin{array}{l}\text { ¿Qué tipo de relación queremos establecer y } \\
\text { mantener nuestro cliente? }\end{array}$ & $\begin{array}{l}\text { Lubricantes Vega tiene la intencionalidad de } \\
\text { establecer una interacción de respeto mutuo, } \\
\text { cordialidad, seriedad, compromiso y transparencia. }\end{array}$ \\
$\begin{array}{ll}\text { ¿Qué relaciones hemos establecido con cada } \\
\text { segmento? }\end{array}$ & $\begin{array}{l}\text { Comerciales por lo regular, aunque con algunos se } \\
\text { han establecido relaciones de amistad por su } \\
\text { fidelidad para con la Pyme }\end{array}$ \\
$\begin{array}{ll}\text { ¿Cuál es su costo? } & \text { No aplicaría, puesto que no se ha adquirido ninguna } \\
\text { sistema o aplicativo tecnológico para tal fin. }\end{array}$ \\
$\begin{array}{ll}\text { ¿Cómo se integran nuestros asociados y } \\
\text { clientes en nuestro modelo de negocio? }\end{array}$ & Como clientes básicamente \\
\hline
\end{tabular}




\section{Relación con los clientes}

- Cliente/Vendedor (Captación, fidelización)

- Atención personalizada (domicilios)

\section{Bloque 5. Fuente De Ingresos}

Este bloque corresponde al fundamento financiero del modelo. Sin ingresos, así el modelo sea excelente teóricamente, no va a poder sobrevivir en la realidad. Aquí en este apartado es necesario relacionar las fuentes de ingreso, tanto los desembolsos emitidos por los clientes por el pago de los productos y servicios, como otras fuentes de entidades externas (inversionistas), o terceros, mediante alianzas estratégicas.

\section{Ilustración 9. Fuente de ingresos}

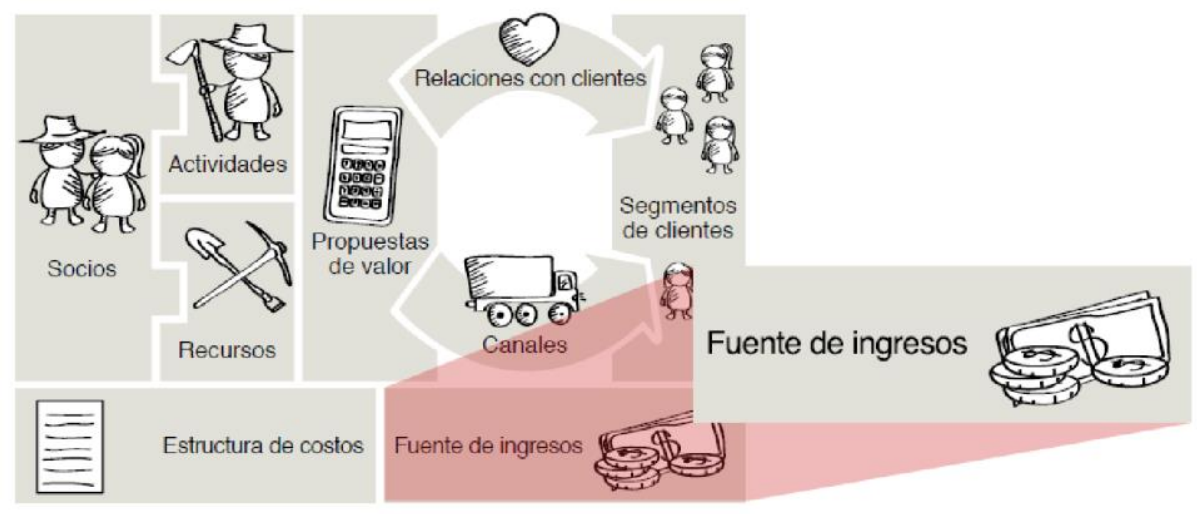

Fuente: Mycoop Colombia

\section{Preguntas relacionadas con sus respectivas respuestas}

\begin{tabular}{ll}
\hline Preguntas & $\begin{array}{l}\text { Respuestas para la Pyme (Lubricantes Vega } \\
\text { S.A.S }\end{array}$ \\
\hline $\begin{array}{l}\text { Qué precio están dispuestos a pagar nuestros } \\
\text { asociados y }\end{array}$ & $\begin{array}{l}\text { De gestar un proceso de asociación o convenio entre } \\
\text { clientes y asociados, se manejaría un descuento de un } \\
\text { cuál nuestros clientes } i \\
\text { caso de los productos que se distribuyen en el local, el } \\
\text { descuento podría manejarse hasta un } 10 \% \text { porque } \\
\text { estos la elasticidad es inelástica para esta clase de } \\
\text { bienes. }\end{array}$ \\
$\begin{array}{l}\text { ¿Cómo pagan o pagarán? } \\
\text { PQué ahora existe solo la modalidad de pago de contado, } \\
\text { pero es necesario ofrecer alternativas como pago con } \\
\text { tarjeta débito o crédito, o chequera. }\end{array}$ \\
\hline
\end{tabular}



mensajes instantáneos por whatsapp o mensajes de texto.

Otra alternativa seria habilitar un espacio lúdicorecreativo para los clientes que tengan que esperar el servicio de lubricación en el local (billar, juegos de sapo, juegos de mesa, cafetería, entre otros)

\section{Potenciales fuentes de ingresos}

\begin{tabular}{ll}
\hline Fuente de ingreso & Observación \\
\hline Venta & Es la fuente principal de ingresos (directa) \\
Cobro por uso & No aplica \\
Cuota de suscripción & No aplica \\
Préstamo, alquiler o leasing & No aplica \\
Concesión de licencias/franquicias & Se podría tener franquicias en poblaciones cercanas a \\
& $\begin{array}{l}\text { Palmira, como Amaime, El Bolo-Santa Helena, Rozo, } \\
\text { entre otros. }\end{array}$ \\
Gastos de corretaje (porcentaje de la venta) & No aplica \\
Publicidad & $\begin{array}{l}\text { Podría resultar eficaz tener un espacio visual en los } \\
\text { noticieros regionales (video promocional de 30 } \\
\text { segundos, donde se exhibe el frente del local y de sus } \\
\text { instalaciones } \\
\text { Los aportes serían en partes iguales por cada socio. }\end{array}$ \\
Aporte de asociados & $\begin{array}{l}\text { Se pueden plantear alianzas estratégicas para } \\
\text { formalizar nuevos negocios. }\end{array}$ \\
Benefactores, inversores y padrinos &
\end{tabular}

\section{Bloque 6. Recursos Clave}

El bloque 6 discute interrogantes de los bloques del "cómo lo hago". Los recursos clave se refieren a los activos más importantes para crear y ofrecer la propuesta de valor y que el modelo de negocio funcione en la realidad 


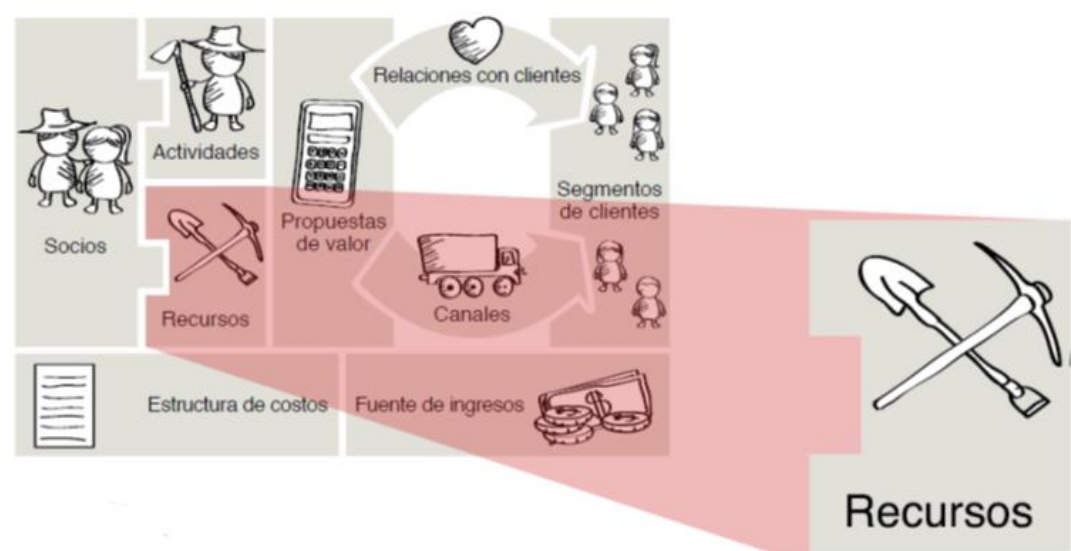

Fuente: Mycoop Colombia

\section{Preguntas relacionadas con sus respectivas respuestas}

\section{Preguntas}

Qué recursos físicos, logísticos, humanos y económicos requiere nuestra propuesta de valor, los canales de distribución, las fuentes de ingreso y la manera de relacionarnos con nuestros asociados y clientes?

\section{Respuestas para la Pyme (Lubricantes Vega S.A.S}

De acuerdo a lo que se requiere para obtener un mayor posicionamiento en la mente del cliente, los recursos idóneos serian:

Recursos físicos: Dos áreas adicionales para poder atender tres automotores en forma simultánea. De igual forma dos máquinas que permitan elaborar sincronización y balanceo (serviteca).

Recursos logísticos: Una estrategia para poder optimizar la gestión de prestación de servicio de lubricación a domicilio, una camioneta con los equipos portátiles adecuados para dicho servicio.

Recursos humanos: Se requerirá de más personal operativo si se amplía el local, al igual que una persona encargada exclusivamente de la gestión comercial, con el fin de identificar nuevos segmentos de mercado.

Recursos económicos: Se requerirá una partida de unos 100 millones para llevar a cabo, los ajustes locativos pertinentes y la adquisición de los equipos y demás dispositivos técnicos.

\section{Bloque 7. Actividades Clave}

Las actividades clave son las acciones más importantes que uno debe hacer, para que su modelo de negocio funcione. 


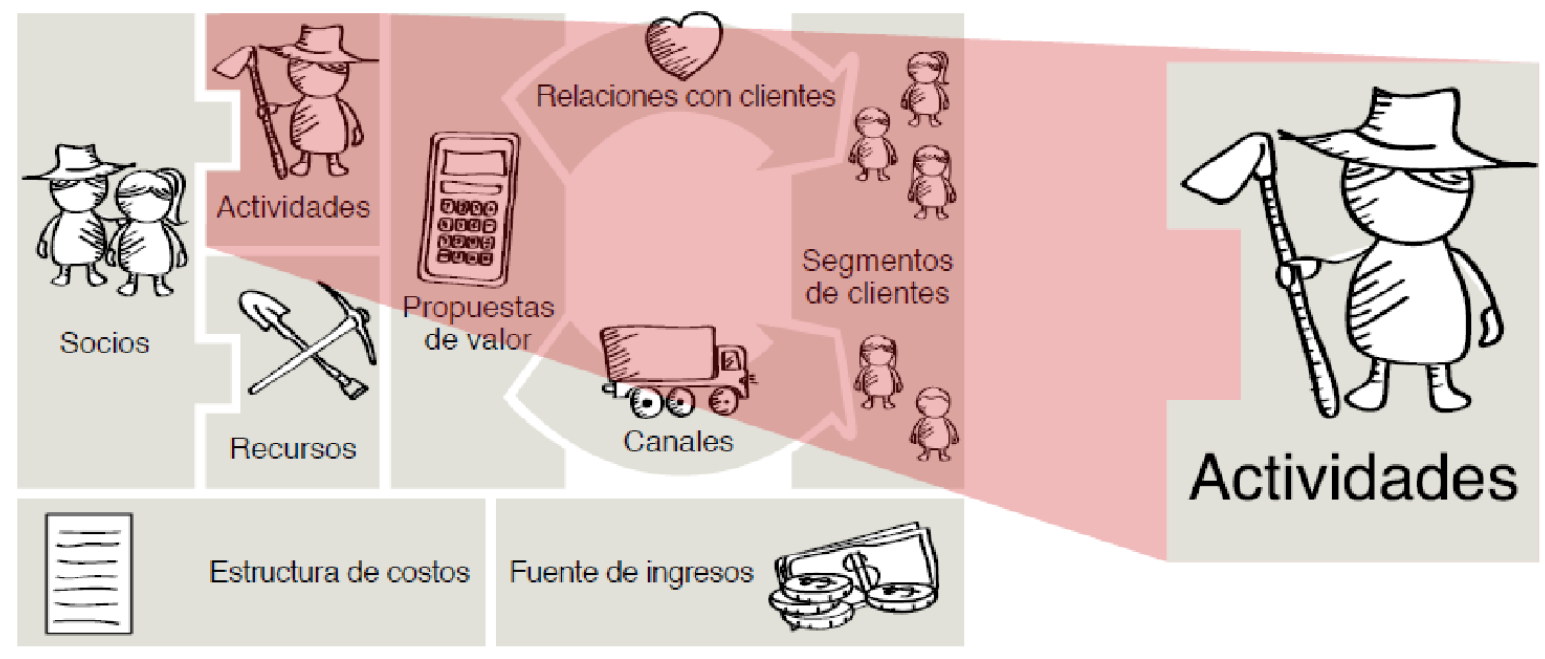

Fuente: Mycoop Colombia

\begin{tabular}{l}
\hline Preguntas \\
\hline ¿Qué actividades clave se requieren realizar \\
para poder hacer nuestra propuesta
\end{tabular}

de valor?

\section{Respuestas para la Pyme (Lubricantes Vega S.A.S}

La propuesta de valor de Lubricantes Vega, es "buena salud para su automotor", en virtud de cumplir esa premisa, se requiere:

- Sostener un surtido con las mejores marcas (conservar la interacción comercial con los proveedores de las marcas más representativas de lubricantes y similares)

- Ampliar la mano de obra calificada para garantizar procesos limpios y de optima calidad

- Propender por ofrecer un servicio oportuno y eficiente (in situ y a domicilio)

- Establecer una políticas de precio razonables consecuentes con la calidad de los productos

- Reformular las estrategias corporativas, de mercadeo y de servicio al cliente

- Llevar a cabo los ajustes locativos que permitan ampliar la bahía operativa

- Adquirir Sistemas de Información. 
canales de distribución?

¿Qué actividades clave requiere la relación con el cliente?

¿Qué actividades clave requiere las fuentes de ingreso? dos; el canal indirecto, local, donde el cliente llega y adquiere el producto y el servicio a domicilio; para el primero lo que se requiere son ajustes locativos en cuanto a cambio de estanterías y modernización de vitrinas y accesorios; y en cuanto al canal del servicio a domicilio se requiere acondicionar los equipos electrónicos de medición de lubricacion al vehículo de la empresa.

Adquirir o diseñar un sistema CRM (Customer Relationship Management) para mejorar la gestión de los clientes

Para tal fin, se puede recurrir a las alianzas estratégicas, o en su defecto idear un plan estratégico para conseguir inyección económica de inversores o abrir la posibilidad jurídica de recibir aportes de nuevos asociados.

\section{Bloque 8. Alianzas Clave (Socios)}

Este bloque hace parte de secuencia final de la herramienta CANVAS, en la cual se procede a estudiar las alianzas claves, esto es, la red de proveedores y socios que contribuyen al funcionamiento del modelo de negocios con el fin de optimizarlo, de reducir riesgos e incertidumbre, adquirir recursos o realizar actividades en conjunto.

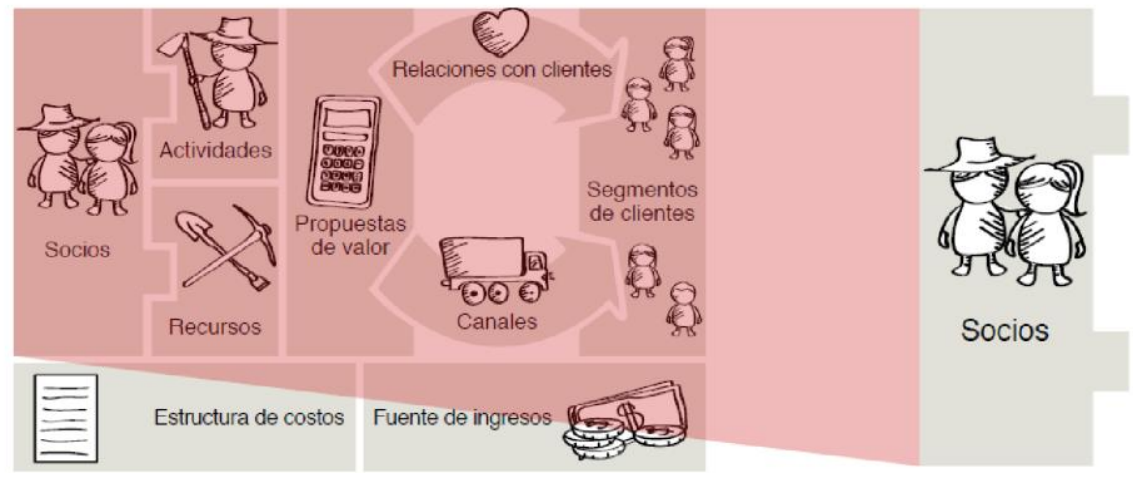

Fuente: Mycoop Colombia 
¿Quiénes son nuestros asociados y socios comerciales clave?

¿Quiénes son nuestros proveedores?

¿Qué recursos adquirimos desde nuestros asociados, socios, comerciales y proveedores terceros?

¿Qué actividades realizan nuestros asociados, socios comerciales

y proveedores?

¿Lo que nos suministran es producido directamente por ellos?
Lubricantes Vega corresponde jurídicamente a una Sociedad de Acciones Simplificadas, con tres accionistas (todos perteneciente a la Familia Vega Gonzalez)

Las empresas manufactureras que tienen relación comercial en calidad de proveedores la conforman principalmente:

- Lubrisol de Colombia

- Mineroil Lubricantes

- Poweroil lubricantes

- Lubricantes BEG

Coexito S.A.S

Existe un Comité de Socios, que aprueban todo lo relacionado con la gestión de compras, aparte de estos, no se obtienen recursos adicionales

Asociados: velar por el sostenimiento financiero de la empresa

Socios comerciales: No aplica

Proveedores: interactuar comercialmente con la empresa, establecer comunicación permanente con el Gerente/Propietario para promover otras referencias, preguntar por el estado de la mercancía, promulgar políticas de precios, etc.

Todos los proveedores anteriormente mencionados, si, pero en el caso de Coexito, ellos también realizan un proceso de distribución, en calidad de mayorista.

Se espera, crear alianzas estratégicas con algunas EDS (Estación de Servicios), como también con medianas empresas transportadoras y manufactureras que operan en el municipio de Palmira, en una relación gana-gana.

\section{Bloque 9. Estructura De Costos}

El bloque final es fundamental porque es necesario determinar los costos más importantes involucrados en mi negocio. 


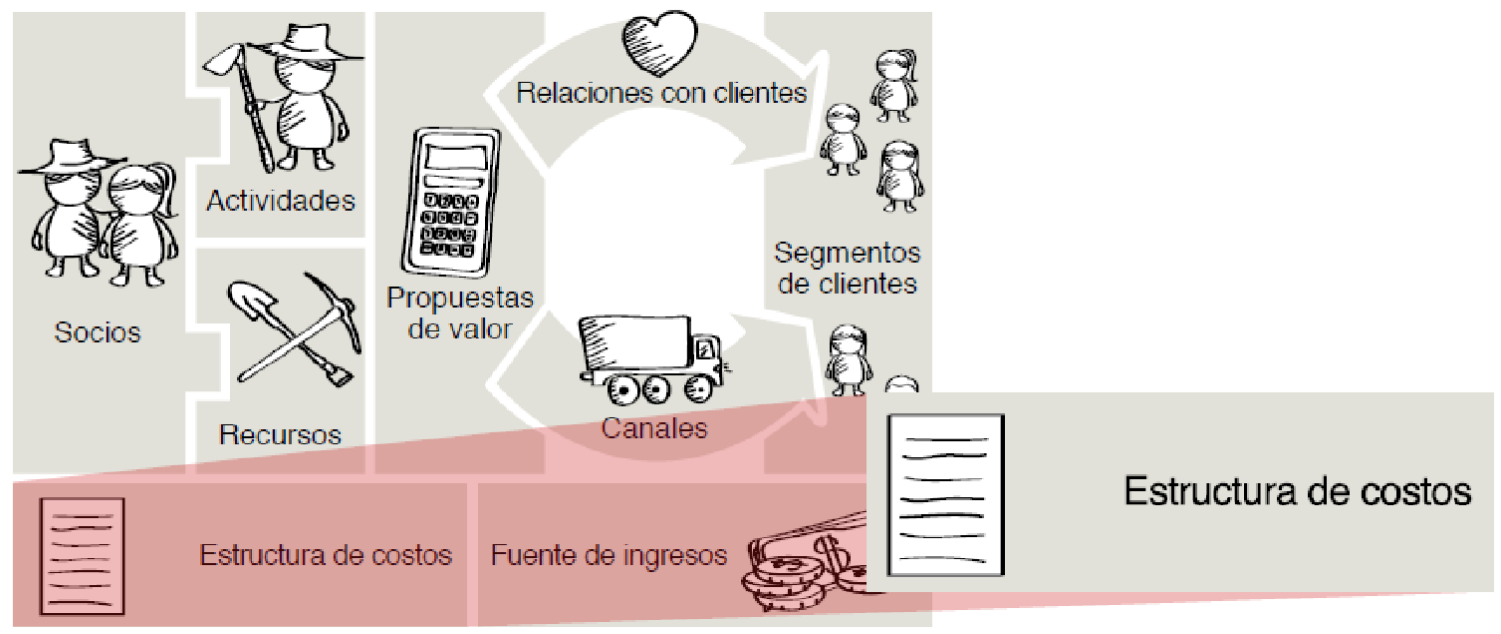

Fuente: Mycoop Colombia

\begin{tabular}{l}
\hline Preguntas \\
\hline ¿Cuáles son los costos y gastos más \\
importantes que se generan en nuestro modelo \\
de negocios?
\end{tabular}

¿Cuáles son los recursos más costosos?

¿Cuáles son las actividades más costosas?

¿Existe alguna manera para que se puedan obviar o disminuir algunos costos?

\section{Respuestas para la Pyme (Lubricantes Vega}

\section{S.A.S}

Costos más representativos:

- Costo de la mercancía a distribuir

- Mano de obra directa

- Impuestos

El recurso humano, y los recursos técnicos para prestar el servicio de lubricación

Las actividades más costosas son aquellas relacionadas con la logística de la prestación del servicio de lubricación a domicilio

Al mejorar la gestión logística de la prestación del servicio a domicilio, se reducirían los gastos por este rubro en un $50 \%$

\section{Resultados y discusión}

Los resultados obtenidos en este proyecto investigativo, evidencian que después de haberse aplicado el Modelo Canvas, el panorama para la Gerencia y asociados de esta microempresa cambia diametralmente, en el sentido en que la didáctica esquematización del negocio en un solo lienzo permite tener siempre presente los ítems a mejorar.

Se confirma la eficacia de esta metodología, al menos potencialmente, puesto que una de las particulares de este modelo, es que los resultados se darán en el plazo 
que las actividades clave se realicen, previo discernimiento de cuáles son, y que se requiere para su desarrollo.

Uno de los elementos diferenciadores de la aplicación de este modelo, es la capacidad sintética que tiene su estructura, en cuanto a la integración de los procesos de la compañía, es decir, el empresario sin llevar a cabo complejos análisis, puede hallar de primera mano, la información visual en un solo lugar (lienzo), y así puede ir tomando decisiones en forma sistemática, con base en los ítems resultantes de cada proceso o bloque.

Lubricantes Vega S.A.S es un negocio rentable, pero no por ello, el propietario puede pensar que ya está todo hecho, y que el objetivo trazado de consolidar la empresa como una organización exitosa, ya se ha cumplido; precisamente el modelo Canvas, lo que posibilita al que lo aplique no solo es garantizarle que el negocio se sostenga, sino que lo haga con un sólido conocimiento, de su realidad corporativo, desde una perspectiva estratégica, que hago, como lo hago, por qué lo hago, para quien lo hago, que me gano con hacerlo asi?...entre otros cuestionamientos.

\section{Conclusiones}

Mediante la aplicación de este modelo Canvas, el propietario pudo identificar mediante el análisis especifico proceso por proceso, qué elementos requiere o puede necesitar para mejorar o en su defecto optimizar la gestión de cada uno de ellos, por ejemplo, analizar cuáles son las actividades que resultan más costosas para la empresa; y tomar decisiones al respecto, no la simple idea que reducir costos por alcanzar mayor margen de utilidad, sino determinar si vale la pena o no seguir ofreciendo "x" o "y" servicio.

\section{Literatura citada}

Matiz, A. (2014). El paso a paso de la construcción de un modelo de negocio basado en el lienzo Canvas. México: Matiz \& Asociados .

Osterwalder, A., \& Pigneur, Y. (2011). Generación de modelos de negocio. Madrid: Deusto S.A. 